\title{
Cambio climático y evaluación de riesgo para el patrimonio cultural
}

\author{
Mauro G. García Santa Cruz, M. Jimena García Santa Cruz, Guillermo R. García | Instituto de Investigación en Arquitectura y \\ Territorio, Facultad de Arquitectura y Diseño, Universidad Católica de La Plata
}

URL de la contribución <www.iaph.es/revistaph/index.php/revistaph/article/view/4988>

El Quinto Informe de Evaluación del IPCC (Grupo Intergubernamental de Expertos sobre Cambio Climático) afirma que desde 1950 se han observado cambios en la atmósfera y el océano debidos al aumento de las concentraciones de gases de efecto invernadero (GEI). También alerta de que las emisiones continuas de GEI causarán un mayor calentamiento y aumentará la probabilidad de impactos severos, generalizados e irreversibles en los ecosistemas (IPCC 2015). Se estima que las actividades humanas han causado un calentamiento global de aproximadamente $1,0^{\circ} \mathrm{C}$ con respecto a los niveles preindustriales. También es probable que el calentamiento global llegue a $1,5^{\circ} \mathrm{C}$ entre 2030 y 2050 si continúa aumentando al ritmo actual. Los riesgos relacionados con el clima dependen de la magnitud y el ritmo del calentamiento, la ubicación geográfica y los niveles de desarrollo y vulnerabilidad, así como de las opciones de adaptación y mitigación que se implementen. Los modelos climáticos prevén un aumento de la temperatura media, aumento de los episodios de calor extremo, aumento de las precipitaciones intensas, además advierten sobre la probabilidad de sequía y de déficits de precipitación en algunas regiones (IPCC 2019). Para mitigar el cambio climático es necesario reducir las emisiones de GEl e implementar medidas de adaptación. En ese sentido, existen estrategias, relacionadas con la conservación del patrimonio cultural y natural, que pueden contribuir con los objetivos propuestos en el Acuerdo de París y en la Agenda 2030 (García Santa Cruz, García Santa Cruz y García 2020; ONU 2015a, 2015b).

El informe El Futuro de Nuestros Pasados: Participación del Patrimonio Cultural en la Acción Climática analiza las intersecciones entre patrimonio cultural, cambio climático y desarrollo sostenible, correlaciona las com- petencias del patrimonio con las prioridades de acción climática, estudia medidas de adaptación y mitigación, analiza pérdidas y daños generados por el cambio climático. El informe concluye que un mejor entendimiento en la forma en que el cambio climático afecta al patrimonio cultural aumentaría la efectividad de las acciones de adaptación necesarias y permitiría generar herramientas de resiliencia para las comunidades (ICOMOS 2019). En diciembre de 2020, durante el quinto aniversario del Acuerdo de París, la 20. ${ }^{a}$ Asamblea General del ICOMOS aprueba la Declaración de Emergencia Climática y Ecológica. La declaración reconoce que el mundo no está cumpliendo los objetivos de París y que ya se están sintiendo los impactos adversos sobre el patrimonio cultural. También reconoce el inmenso potencial del patrimonio cultural para permitir una acción climática inclusiva, transformadora y justa. Pide a la comunidad que trabaje para aprovechar este potencial y salvaguardar el patrimonio de los impactos climáticos adversos, lograr un desarrollo sostenible resiliente al clima y reconocer la equidad y la justicia como dimensiones fundamentales de la acción climática (ICOMOS 2020).

En este contexto, a partir del trabajo colaborativo de ICOMOS Argentina, la Universidad Católica de La Plata, la Iniciativa Patrimonio y Cambio Climático, y la Fundación Ciudad de La Plata, fue posible organizar el curso en línea Cambio climático y evaluación de riesgo para el patrimonio cultural ${ }^{1}$.

Como actividad integradora de este curso virtual, los estudiantes y profesionales debieron realizar la evaluación de los riesgos que pueden afectar el patrimonio de una ciudad determinada. Para este estudio se consideraron los riesgos de origen natural y antrópico (Carrión 


\begin{tabular}{|c|c|c|c|c|c|c|c|c|c|c|c|c|c|c|c|c|}
\hline Origen & Riesgo analizado & C1 & C2 & C3 & C4 & C5 & C6 & C7 & C8 & C9 & C10 C & C11 & C12 & C13 & C14 & C15 \\
\hline \multirow{10}{*}{$\begin{array}{l}\bar{\pi} \\
\frac{\sqrt{0}}{2} \\
\frac{\pi}{2}\end{array}$} & Terremotos & 7 & 9 & 9 & 2 & 5 & 0 & 0 & 0 & 1 & 0 & 0 & 2 & 2 & 10 & 10 \\
\hline & Deslizamientos de tierra & 5 & 5 & 9 & 3 & 3 & 0 & 0 & 0 & 1 & 5 & 3 & 1 & 2 & 7 & 5 \\
\hline & Erupciones volcánicas & 6 & 6 & 0 & 0 & 0 & 0 & 0 & 0 & 0 & 0 & 0 & 0 & 0 & 0 & 0 \\
\hline & Maremotos & 4 & 7 & 0 & 5 & 0 & 0 & 0 & 0 & 0 & 0 & 7 & 2 & 0 & 10 & 7 \\
\hline & Huracanes & 10 & 0 & 0 & 8 & 0 & 8 & 8 & 2 & 2 & 0 & 4 & 2 & 0 & 0 & 0 \\
\hline & Inundaciones & 10 & 7 & 7 & 9 & 3 & 7 & 8 & 7 & 4 & 2 & 5 & 6 & 6 & 3 & 8 \\
\hline & Tornados & 5 & 0 & 0 & 6 & 2 & 5 & 0 & 4 & 2 & 3 & 2 & 2 & 4 & 0 & 0 \\
\hline & Nevadas & 0 & 4 & 6 & 7 & 0 & 6 & 0 & 2 & 0 & 9 & 0 & 0 & 2 & 1 & 0 \\
\hline & Lluvias & 10 & 8 & 8 & 9 & 4 & 8 & 8 & 8 & 3 & 8 & 9 & 5 & 8 & 8 & 5 \\
\hline & Incendios & 8 & 8 & 6 & 6 & 5 & 9 & 0 & 5 & 2 & 6 & 8 & 6 & 0 & 10 & 10 \\
\hline \multirow{10}{*}{$\begin{array}{l}\frac{0}{0} \\
\frac{0}{0} \\
\frac{2}{2} \\
\frac{1}{4}\end{array}$} & Pandemias & 10 & 5 & 8 & 6 & 6 & 7 & 5 & 7 & 8 & 10 & 7 & 5 & 6 & 5 & 5 \\
\hline & Negligencia & 8 & 6 & 7 & 8 & 4 & 7 & 6 & 5 & 7 & 9 & 6 & 8 & 9 & 6 & 9 \\
\hline & Errores técnicos & 8 & 5 & 9 & 8 & 3 & 5 & 4 & 5 & 6 & 9 & 6 & 7 & 7 & 3 & 6 \\
\hline & Falta de mantenimiento & 8 & 6 & 10 & 7 & 3 & 7 & 6 & 7 & 6 & 10 & 5 & 9 & 10 & 6 & 10 \\
\hline & Averías & 8 & 5 & 8 & 7 & 4 & 4 & 4 & 6 & 7 & 5 & 3 & 7 & 7 & 0 & 9 \\
\hline & Bélicos & 2 & 3 & 2 & 6 & 0 & 6 & 0 & 2 & 0 & 0 & 0 & 5 & 6 & 0 & 2 \\
\hline & Sabotajes & 2 & 3 & 8 & 7 & 0 & 6 & 0 & 4 & 3 & 0 & 6 & 6 & 8 & 0 & 0 \\
\hline & Vandalismo & 6 & 6 & 6 & 8 & 4 & 6 & 2 & 8 & 5 & 4 & 2 & 6 & 8 & 0 & 7 \\
\hline & Terrorismo & 4 & 0 & 6 & 9 & 5 & 8 & 0 & 4 & 0 & 0 & 0 & 5 & 0 & 0 & 0 \\
\hline & Psicopáticos & 4 & 0 & 6 & 7 & 2 & 7 & 0 & 6 & 2 & 4 & 3 & 4 & 5 & 0 & 0 \\
\hline \multirow{20}{*}{ 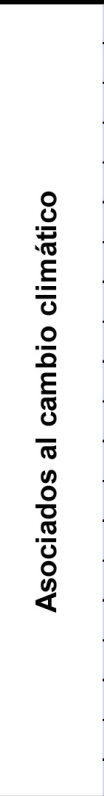 } & Aumento de la temperatura & 7 & 7 & 2 & 10 & 3 & 8 & 8 & 8 & 8 & 5 & 6 & 7 & 9 & 8 & 5 \\
\hline & Cambio en los ciclos de hielo-deshielo & 2 & 6 & 2 & 10 & 0 & 4 & 0 & 0 & 0 & 6 & 6 & 0 & 0 & 1 & 0 \\
\hline & Deshielo del permafrost & 0 & 4 & 2 & 10 & 0 & 0 & 0 & 0 & 0 & 0 & 6 & 0 & 0 & 0 & 0 \\
\hline & Aumento del contenido de vapor de agua en el aire & 7 & 7 & 4 & 10 & 4 & 7 & 6 & 6 & 9 & 6 & 2 & 7 & 6 & 0 & 7 \\
\hline & Aumento del viento & 10 & 6 & 8 & 8 & 2 & 8 & 0 & 6 & 6 & 5 & 8 & 7 & 5 & 0 & 7 \\
\hline & Incendios forestales influenciados por el clima & 4 & 9 & 7 & 0 & 0 & 9 & 0 & 5 & 8 & 0 & 8 & 6 & 0 & 10 & 10 \\
\hline & Cambios en la estacionalidad y la fenología & 6 & 7 & 4 & 7 & 6 & 4 & 0 & 4 & 8 & 5 & 4 & 7 & 9 & 9 & 5 \\
\hline & Propagación de especies invasoras y plagas & 4 & 4 & 4 & 7 & 2 & 9 & 2 & 6 & 4 & 7 & 8 & 6 & 6 & 10 & 7 \\
\hline & Cambios en rango, distribución y poblaciones de especies & 0 & 4 & 4 & 6 & 0 & 4 & 0 & 6 & 2 & 7 & 4 & 6 & 4 & 10 & 7 \\
\hline & Menos precipitación o sequía & 5 & 10 & 4 & 3 & 2 & 8 & 4 & 4 & 5 & 2 & 2 & 7 & 8 & 10 & 6 \\
\hline & Desertificación & 0 & 10 & 4 & 0 & 0 & 0 & 4 & 4 & 2 & 0 & 2 & 0 & 0 & 0 & 7 \\
\hline & Aumento precipitación y eventos de lluvia más intensos & 10 & 8 & 8 & 9 & 3 & 9 & 8 & 8 & 7 & 7 & 8 & 7 & 8 & 0 & 4 \\
\hline & Eventos agudos de inundaciones costeras & 10 & 8 & 0 & 9 & 2 & 8 & 8 & 6 & 4 & 0 & 9 & 4 & 8 & 0 & 6 \\
\hline & Aumento de tormentas (incluidos huracanes y ciclones) & 10 & 2 & 0 & 9 & 0 & 8 & 8 & 8 & 5 & 5 & 6 & 4 & 7 & 0 & 0 \\
\hline & Aumento de la erosión costera & 10 & 2 & 0 & 9 & 0 & 4 & 0 & 6 & 5 & 0 & 7 & 2 & 7 & 0 & 7 \\
\hline & Aumento del nivel freático & 6 & 2 & 6 & 9 & 4 & 7 & 2 & 4 & 5 & 7 & 5 & 4 & 5 & 0 & 6 \\
\hline & Intrusión de agua salada & 6 & 6 & 0 & 9 & 0 & 6 & 0 & 2 & 5 & 0 & 6 & 4 & 0 & 8 & 1 \\
\hline & Acidificación del océano (por aumento de CO2) & 9 & 2 & 0 & 10 & 0 & 7 & 0 & 0 & 7 & 0 & 6 & 6 & 0 & 0 & 6 \\
\hline & Contaminación (factor de estrés secundario) & 8 & 5 & 6 & 9 & 5 & 9 & 2 & 6 & 9 & 5 & 5 & 8 & 10 & 10 & 5 \\
\hline & Desarrollo impulsado por el clima & 8 & 4 & 6 & 2 & 2 & 7 & 0 & 2 & 2 & 3 & 8 & 6 & 6 & 0 & 4 \\
\hline
\end{tabular}

Matriz de riesgos para el patrimonio, estudio realizado sobre quince ciudades | fuente Construcción de la matriz y análisis de datos por M.G. García Santa Cruz, a partir de los estudios de caso analizados por estudiantes y profesionales de la edición 2021 del curso virtual 
Gútiez 2015), además de los riesgos asociados al cambio climático (ICOMOS 2019), que podrían afectar al patrimonio de esa ciudad. Se realizó una investigación sobre los eventos que habían afectado a la ciudad seleccionada como caso de estudio en los últimos cien años. La evaluación de riesgos considera diez riesgos de origen natural, diez riesgos de origen antrópico y veinte riesgos asociados al cambio climático. Luego determinaron la posibilidad de ocurrencia de los riesgos analizados, desde sin existencia (0) o muy baja (1), hasta muy alta (5), y el posible impacto que estos riesgos tendrían para el patrimonio de esa ciudad, desde sin impacto (0) o muy pequeño (1), hasta muy grave (5). Finalmente, considerando la posibilidad de que el riesgo acontezca y el impacto asociado, se determinaron las correspondientes "magnitudes de riesgo", desde sin impacto (0) o baja prioridad (2), hasta catastrófica (10). La "matriz de riesgos" compila los resultados para los siguientes casos de estudio": Bidgetown (Barbados); Cusco (Perú); Dublín (Irlanda); El Cairo (Egipto); La Lima (Honduras); La Plata y Rosario (Argentina); Lisboa (Portugal); Montrose (Estados Unidos); Niteroi y Río de Janeiro (Brasil); Concepción, Valdivia y Valparaíso (Chile).

Finalmente, considerando la sumatoria de las magnitudes de los riesgos analizados para cada uno de los casos de estudio, se determina el porcentaje de los impactos relacionados a los riesgos de origen natural, riesgos de origen antrópico y riesgos asociados al cambio climático. Este análisis permite indicar que el impacto de los riesgos asociados al cambio climático representa entre el $40 \%$ y el $60 \%$ de los impactos totales, dependiendo del caso de estudio analizado. Considerando los resultados, cabe destacar la importancia de la implementación de estrategias de adaptación y mitigación que permitan reducir los riesgos asociados al cambio climático. En ese sentido, es posible implementar medidas de adaptación y mitigación frente al cambio climático basadas en la protección, restauración y conservación del patrimonio.

El documento El futuro de nuestros pasados propone una serie de estrategias de adaptación del patrimonio a

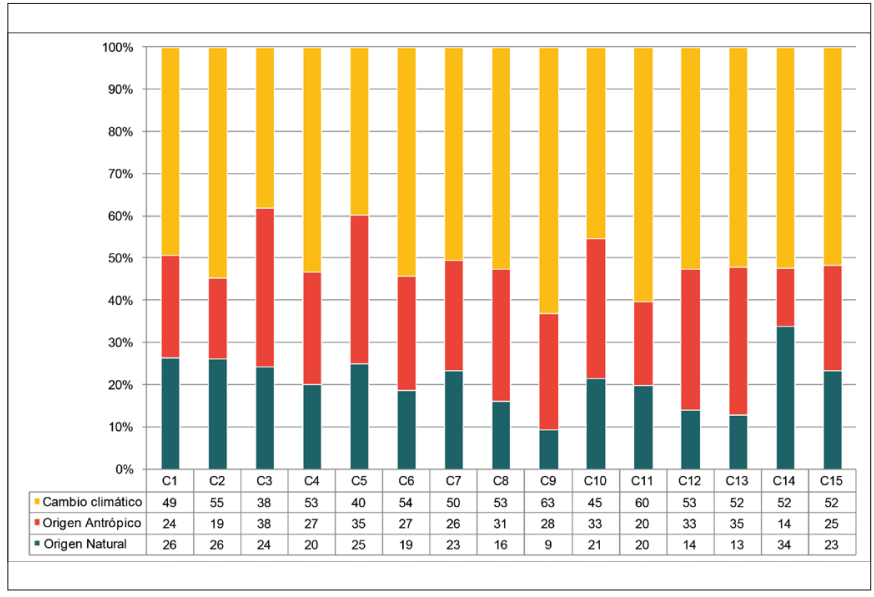

Porcentaje de impactos relacionados a riesgos de origen natural, antrópico y cambio climático | fuente Gráfico y análisis de datos por M.G. García Santa Cruz, a partir de los estudios de caso analizados por estudiantes y profesionales de la edición 2021 del curso virtual

Ios efectos del cambio climático, organizadas en tres grupos: conocimiento y comprensión; planificación e implementación; oportunidades, limitaciones y desafíos. En el primer grupo se encuentran las estrategias basadas en valores y centradas en las personas, recopilación de datos del patrimonio, monitoreo del patrimonio, ejemplos de buenas prácticas. En el segundo grupo se asocian las estrategias vinculadas con la reducción del riesgo de desastres, planificación de la adaptación, adaptación dentro de políticas más amplias. El último grupo lo integran las estrategias relacionadas con la gestión del cambio, oportunidades, incertidumbre, costes y beneficios, sostenibilidad (ICOMOS 2019).

El patrimonio cultural, con sus valores tangibles e intangibles, interactúa directa e indirectamente con el Acuerdo de París (ICOMOS 2019). La descarbonización es clave para mitigar las emisiones de gases de efecto invernadero. Para limitar el calentamiento a $1,5^{\circ} \mathrm{C}$ las emisiones anuales deben ser aproximadamente la mitad de su tasa actual para 2030 y las emisiones netas de gases de efecto invernadero, en promedio, deberán reducirse a cero para 2050 (ONU 2015a). El documento El futuro de nuestros pasados propone también una serie de estrategias de mitigación del cambio climático organizadas en siete grupos: vivir sosteniblemente; mitigación a través 
de medidas centradas en la demanda; mitigación a través de medidas en otros sectores; patrimonio y eliminación de $\mathrm{CO}_{2}$; mitigación a través de medidas centradas en la oferta; turismo cultural; y el patrimonio como motor de la mitigación (ICOMOS 2019).

En el documental Adaptación y Mitigación basadas en el patrimonio 3 (FOPS 2020a, 2020b, 2020c) profesionales de Argentina, Chile, España, Irlanda, México, Nueva Zelanda, Perú, Reino Unido y Estados Unidos reflexionan sobre la emergencia climática global y proponen medidas de adaptación y mitigación frente al cambio climático basadas en el patrimonio.

\section{Agradecimientos}

Agradecemos a las instituciones que organizaron y auspiciaron las tres ediciones del curso Cambio climático y evaluación de riesgo para el patrimonio cultural. A los profesores del curso Guillermo R. García, M. Gabriela Santibañez, Mauro García Santa Cruz, Jimena García Santa Cruz, y profesores invitados Pedro Delheye, Walter Di Santo, Miguel Iwadare, Toshiyuki Kono, Peter Cox, Andrew Potts, Christopher Marrion, Stacy Vallis. A los estudiantes y profesionales que participaron de las tres ediciones del curso, especialmente a quienes realizaron los estudios de caso presentados en este artículo.

\section{NOTAS}

1. El curso contó con el auspicio de Climate Heritage Network (CHN); UNESCO Oficina Regional de Ciencias para América Latina y el Caribe; International Council on Monuments and Sites (ICOMOS); Climate Change and Heritage Working Group (ICOMOS CCHWG); International Committee on Risk Preparedness (ICOMOS ICORP); International Emerging Professionals Working Group (ICOMOS EPWG); Asociación de Directores de Museos de la República Argentina (ADiMRA); Facultad de Arquitectura y Diseño, Universidad Católica de La Plata; Universidad de Congreso; Universidad de Mendoza; Instituto de Investigación en Turismo e Identidad (Universidad de Cuyo). La edición 2019 se realizó de forma presencial en el Museo de Arte Contemporáneo Beato Angélico (Universidad Católica de La Plata), contó con el patrocinio del Fondo Nacional de las Artes y la participación de cincuenta profesionales de la región de La Plata y Buenos Aires, en Argentina. Las ediciones 2020 y 2021 se realizaron de forma virtual y contaron con la participación de profesores de ICOMOS Argentina y profesores invitados de ICOMOS Japón, Irlanda, Estados Unidos, México y Nueva Zelanda. Estas ediciones virtuales contaron en total con la participación de ochenta profesionales que residen en Alemania, Argentina, Australia, Barbados, Bolivia, Brasil, Canadá, Chile, CoIombia, Costa Rica, Estados Unidos, Filipinas, Grecia, Honduras, Inglaterra, Irlanda, México, Perú, Portugal, Suecia y Turquía.

2. Los casos de estudio fueron analizados por Andrea Richards Cummins, Brian Michael Lione, Carmen Fajardo Cardona, Diana Santander Cjuno, Florentina Spiazzi, Francisca Reyes San Martín, Helena Martelo, Javiera Errázuriz, Karin Weil González, Layse Souza Costa, Michelle Tizuka, Petra Strakendal, Rocío Ramírez Zúñiga, Teresa Sweeney Meade y Valentina Maurino.

3. Este documental es resultado de la edición 2020 del curso virtual Cambio climático y evaluación de riesgo para el patrimonio cultural. Se estrenó en noviembre de 2020 en el marco de Culture by Climate 2020, un foro mundial para las artes, la cultura y la acción climática basada en el patrimonio organizado por Climate Heritage Network (http://climateheritage.org/). El documental puede verse en el canal de YouTube del Foro Patrimonio Sustentable (https://www.youtube.com/c/ForoPatrimonioSustentable), el mismo dispone de subtítulos en español, portugués e inglés. Además se puede consultar más información sobre las actividades mencionadas en este artículo en el Blog del Foro Patrimonio Sustentable (https://foropatrimoniosustentable.blogspot.com/) y en las redes sociales: Twitter @pacc_fops (https://twitter. com/pacc_fops), Facebook @pacc.fops (https://www.facebook.com/pacc.fops) e Instagram @pacc.fops (https:// www.instagram.com/pacc.fops/). 


\section{BIBLIOGRAFÍA}

- Carrión Gútiez, A. (coord.) (2015) Plan Nacional de Emergencias y Gestión de Riesgos en Patrimonio Cultural. Madrid: Secretaría General Técnica, Ministerio de Educación, Cultura y Deporte. Disponible en: https://www.libreria. culturaydeporte.gob.es/libro/plan-nacional-de-emergenciasy-gestion-de-riesgos-en-patrimonio-cultural_3255/ [Consulta: 14/07/2021]

- FOPS [Foro Patrimonio Sustentable] (2020a) Adaptación y Mitigación basadas en el patrimonio (Tráiler del documental). Disponible en: https://youtu.be/xTrLEgcdn7c [Consulta: 14/07/2021]

- FOPS [Foro Patrimonio Sustentable] (2020b) Adaptación y Mitigación basadas en el patrimonio. Parte 1: Emergencia climática global. Disponible en: https://youtu.be/DaHbD4KmNU [Consulta: 14/07/2021]

- FOPS [Foro Patrimonio Sustentable] (2020c) Adaptación y Mitigación basadas en el patrimonio. Parte 2: Adaptación y mitigación. Disponible en: https://youtu.be/cEkIUGOzW_o [Consulta: 14/07/2021]

- García Santa Cruz, M.G., García Santa Cruz, M.J. y García, G.R. (2020) A conservação do patrimônio cultural e natural como estratégia de mitigação das mudanças climáticas. Revista Fórum Patrimônio: Ambiente Construído e Patrimônio Sustentável, vol. 11 n. ${ }^{\circ}$ 1. Disponible en: https://periodicos. ufmg.br/index.php/forumpatrimo/article/view/34036 [Consulta: 15/07/2021]

- ICOMOS [Consejo Internacional de Monumentos y Sitios] (2019) The Future of Our Pasts: Engaging Cultural Heritage in Climate Action. Disponible en: https://indd.adobe.com/ view/a9a551e3-3b23-4127-99fd-a7a80d91a29e [Consulta: 15/07/2021]

- ICOMOS [Consejo Internacional de Monumentos y Sitios] (2020) Resolution 20GA/15-Cultural Heritage and the Climate Emergency. Disponible en: https://www.icomos.org/images/ DOCUMENTS/Secretariat/2020/Cultural_Heritage_and_the Climate_Emergency-Resolution_20GA_15_.pdf [Consulta: 15/07/2021]

- IPCC [Grupo Intergubernamental de Expertos sobre el Cambio Climático] (2015) Cambio climático 2014. Informe de síntesis. Disponible en: https://www.ipcc.ch/site/assets/ uploads/2018/02/SYR_AR5_FINAL_full_es.pdf [Consulta: 14/07/2021]

- IPCC [Grupo Intergubernamental de Expertos sobre el Cambio Climático] (2019) Calentamiento global de $1,5{ }^{\circ} \mathrm{C}$. Resumen para responsables de políticas. Disponible en: https://www.ipcc.ch/site/assets/uploads/sites/2/2019/09/IPCCSpecial-Report-1.5-SPM_es.pdf [Consulta: 14/07/2021]

- ONU [Organización de las Naciones Unidas] (2015a)
Acuerdo de París. Disponible en: https://unfccc.int/sites/default/ files/spanish_paris_agreement.pdf [Consulta: 14/07/2021]

- ONU [Organización de las Naciones Unidas] (2015b) Transformar nuestro mundo: la Agenda 2030 para el Desarrollo Sostenible (Resolución aprobada por la Asamblea General el 25 de septiembre de 2015). Disponible en: https://unctad. org/system/files/official-document/ares70d1_es.pdf [Consulta: 14/07/2021] 